\title{
Functionalization of arrays of silica nanochannels by post-condensation
}

Nicola Zucchetto and Dominik Brühwiler*

Institute of Chemistry and Biotechnology

Zürich University of Applied Sciences

CH-8820 Wädenswil, Switzerland

* Corresponding author: dominik.bruehwiler@zhaw.ch

Dalton Trans. 45 (2016) 14363 - 14369. 


\section{Abstract}

Functionalized arrays of silica nanochannels (ASNCs) were prepared by post-condensation of aminofunctional alkoxysilanes (NH-silanes). The method of post-condensation combines postsynthetic grafting and co-condensation - the classical pathways for the functionalization of mesoporous silica - to afford a convenient one-pot reaction in aqueous medium. The structure of the $\mathrm{NH}$-silanes determines the distribution of the surface-bound groups in the ASNCs. Accumulation at the channel entrances was observed by confocal laser scanning microscopy in the case of sterically hindered $\mathrm{NH}$-silanes, whereas smaller $\mathrm{NH}$-silanes led to a more uniform distribution. In all cases, the channels remained accessible for additional postsynthetic treatment or introduction of guests after extraction of the structure-directing agent. Particle size and morphology, as well as pore size and degree of ordering of the aminofunctionalized ASNCs were comparable to pristine ASNCs. 


\section{Introduction}

Ordered mesoporous silica has received increasing attention in the fields of catalysis, ${ }^{1-3}$ sensors,${ }^{4}$ drug delivery, ${ }^{5}$ and adsorption ${ }^{6}$ due to the large variety of customization options in terms of pore size, pore system dimensionality, particle size, particle morphology, and surface functional groups.

The introduction of functional groups into a mesoporous silica framework is usually achieved by postsynthetic treatment (grafting) or by co-condensation. ${ }^{7}$ Advantages and disadvantages of these methods have been studied extensively. Postsynthetic functionalization introduces the functional groups after the formation of the mesoporous silica particles. The particle size and shape are thus preserved. While this method is not ideal for achieving uniform distributions of functional groups, distinctively nonuniform distributions, such as a selective functionalization of the external particle surface, are possible. $^{8-12}$ Co-condensation is the method of choice when a uniform distribution of the functional groups is desired. In co-condensation, functional groups are incorporated into the silica framework during the formation of the mesoporous structure, typically via the respective alkoxysilanes. Contrary to postsynthetic functionalization, which often uses an organic solvent for the functionalization step, cocondensation relies on functionalization in aqueous medium. The presence of functional alkoxysilanes in the reaction mixture can, however, affect the outcome of the synthesis in terms of particle size, particle morphology, and degree of ordering. ${ }^{13-19}$ High levels of functionalization generally result in a loss of ordering and in increasingly broad pore size distributions.

Arrays of silica nanochannels (ASNCs) are a MCM-41 type material featuring well-defined hexagonal prismatic particles with the channel (pore) entrances located on the base surfaces, as schematically shown in Fig. $1 .^{10,20-23}$ With this highly ordered structure, ASNCs can be considered as the mesoporous analogue to hexagonal zeolites such as zeolite L (LTL framework) ${ }^{24-26}$ or AlPO $_{4}-5$ (AFI framework). ${ }^{27-29}$ Zeolites of this type have been used as host materials for the supramolecular organization of guest 
molecules. ${ }^{30}$ The respective host-guest composites can exhibit fascinating photonic properties, including efficient Förster resonance energy transfer (FRET), ${ }^{31}$ lasing, $^{32}$ or second harmonic generation. ${ }^{33}$

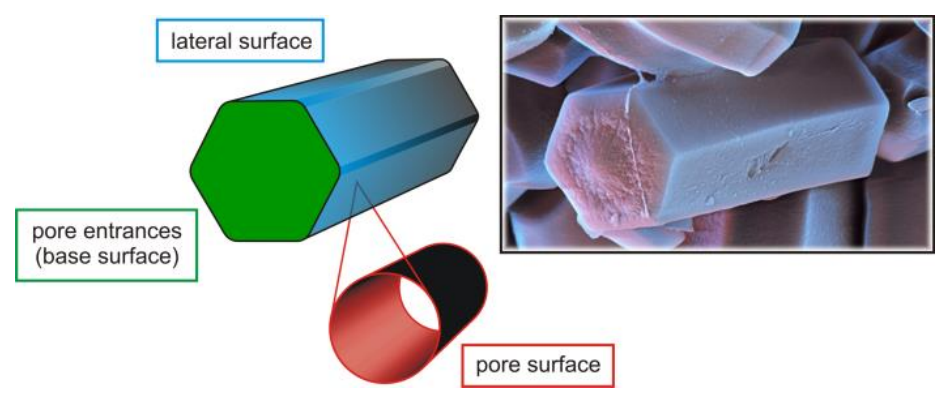

Fig. 1. Illustration of the different surface regions of ASNCs. The particle shown in the scanning electron microscopy image has a length of approximately $5 \mu \mathrm{m}$. The average pore (nanochannel) diameter is typically in the range of 2.5 to $4 \mathrm{~nm}$.

The functionalization of ASNCs has so far only been achieved by postsynthetic treatment, ${ }^{10,22,34}$ as the particle morphology is very sensitive to the conditions during the condensation of the silica framework. The selective functionalization of the different surface regions of ASNCs (Fig. 1) is challenging. The external surface (base and lateral surfaces) has been functionalized by a postsynthetic reaction with a sterically hindered amino-functional trialkoxysilane in a nonpolar solvent. ${ }^{10}$

In order to allow for a one-pot reaction in aqueous medium, we have developed a method that combines postsynthetic treatment with co-condensation. In this so-called post-condensation pathway, the functional alkoxysilanes are introduced into the ASNCs formation process after stabilization of the particle morphology. Primary and secondary amino-functional alkoxysilanes (NH-silanes) were chosen as model substances, as they allow convenient coupling to fluorescent labels for imaging by confocal laser scanning microscopy (CLSM). 


\section{Experimental section}

Materials. Hexadecyltrimethylammonium bromide (CTAB, for molecular biology, $\geq 99 \%), \mathrm{CaCl}_{2}$ ( $\geq 97.0 \%$ ), concentrated hydrochloric acid (puriss p.a., $\geq 32 \%$ ), tetraethoxysilane (TEOS, $99.999 \%$ ), 3aminopropyltrimethoxysilane (APTMS, $99 \%), \quad$ [3-(2-aminoethylamino)propyl]trimethoxysilane (AEAPTMS, $95 \%)$, and $\mathrm{N}^{1}$-(3-trimethoxysilylpropyl)diethylenetriamine (TMPTA, technical grade) were obtained from Sigma-Aldrich. 3-Aminopropyldiisopropylethoxysilane (APDIPES, 97 \%), bis(triethoxysilylpropyl)amine (BTESPA, $95 \%$ ), and 3-aminopropyltris(methoxyethoxyethoxy)silane (APTMEES, $95 \%$ ) were obtained from $A B C R$. The fluorescent labels fluorescein isothiocyanate (FITC, isomer I, $\geq 90 \%$ ) and Texas Red sulfonyl chloride (TR, technical grade) were obtained from Sigma-Aldrich. All chemicals were used as received.

Synthesis of amino-functionalized ASNCs. CTAB $(4.0 \mathrm{~g}, 11 \mathrm{mmol})$ and $\mathrm{CaCl}_{2}(1.50 \mathrm{~g}, 13.5 \mathrm{mmol})$ were dissolved in a mixture of doubly distilled $\mathrm{H}_{2} \mathrm{O}(76 \mathrm{~mL}, 4.2 \mathrm{~mol})$ and concentrated hydrochloric acid ( $\geq 32 \%, 60 \mathrm{~mL}, 0.62 \mathrm{~mol}$ ) by stirring for $15 \mathrm{~min}$ in a polypropylene beaker. The solution was subsequently cooled in an ice bath for 15 min under quiescent conditions, followed by the slow addition of cold TEOS $\left(4{ }^{\circ} \mathrm{C}, 2.0 \mathrm{~mL}, 9 \mathrm{mmol}\right)$ and further stirring for $30 \mathrm{~s}$. The resulting mixture was kept at $0{ }^{\circ} \mathrm{C}$ under quiescent conditions for $3 \mathrm{~h}$. After this period, the solution was stirred for 1 min while adding the desired amount of $\mathrm{NH}$-silane and subsequently kept at $0{ }^{\circ} \mathrm{C}$ under quiescent conditions for $1 \mathrm{~h}$. The product was filtered off and dried at $80^{\circ} \mathrm{C}$. In the case of samples prepared by the classical cocondensation route, the $\mathrm{NH}$-silanes were added with TEOS at the start of the synthesis. The amount of $\mathrm{NH}$-silane was calculated using two different $\mathrm{NH}$-silane/TEOS ratios: 0.1 and 0.3 .

For removing the structure-directing agent (SDA), the product was first heated at $150{ }^{\circ} \mathrm{C}$ for $10 \mathrm{~h}$ and then extracted by stirring a $100 \mathrm{mg}$ amount in a solution of ethanol $(20 \mathrm{~mL})$ and ammonium nitrate (80 mg) at $60{ }^{\circ} \mathrm{C}$ for $15 \mathrm{~min}$, followed by centrifugation. After repeating this extraction step twice, the product was washed with ethanol $(20 \mathrm{~mL})$ and dried at $80^{\circ} \mathrm{C}$ overnight. 
Fluorescent labelling. The samples were labeled by stirring ( $16 \mathrm{~h}$ at room temperature) the aminofunctionalized ASNCs $(50 \mathrm{mg})$ in absolute ethanol $(5 \mathrm{~mL})$ containing FITC (2 mg). After centrifugation, the labeled samples were washed with absolute ethanol until the washing solution became colorless. The samples were dried at $80^{\circ} \mathrm{C}$.

To test the accessibility for pore surface functionalization, extracted FITC-labeled samples (100 mg) were dispersed in absolute ethanol $(5 \mathrm{~mL})$ and APTMS $(2 \mu \mathrm{L})$ was added. After the mixture had been stirred for 10 min at room temperature, the solid was recovered by centrifugation and cured in an oven at $80^{\circ} \mathrm{C}$ for $6 \mathrm{~h}$. TR (1 mg) was added to the dried powder $(50 \mathrm{mg})$ with subsequent stirring in absolute ethanol for $16 \mathrm{~h}$ at room temperature. After centrifugation, the sample was washed repeatedly with absolute ethanol until the washing solution became colorless.

Physical measurements. Nitrogen sorption isotherms were measured at $77 \mathrm{~K}$ with a Quantachrome Autosorb iQ MP. Pore size distributions and pore diameters $d_{\mathrm{DFT}}$ were calculated by non-local density functional theory (NLDFT, silica with cylindrical pores, ASiQwin, version 3.01). ${ }^{35}$ Surface areas $S_{\text {BET }}$ were determined by multipoint BET. ${ }^{36}$ Total pore volumes $V_{\text {tot }}$ were derived from the amount of adsorbed nitrogen at a relative pressure of $p / p_{0}=0.95$. Adsorption isotherms were used for all calculations. Scanning electron microscopy (SEM) images were taken with a FEI Phenom Pro. CLSM was performed with an Olympus BX60 microscope equipped with a FluoView FV300 confocal unit and excitation at 488 or $543.5 \mathrm{~nm}$. Powder X-ray diffraction (XRD) patterns were recorded on a STOE STADI P diffractometer with $\mathrm{CuK \alpha}_{1}$ radiation. 


\section{Results and discussion}

Morphology. The investigated amino-functional alkoxysilanes, in the following termed as $\mathrm{NH}$-silanes, are shown in Fig. 2. A conventional co-condensation approach with TEOS and NH-silanes under the synthesis conditions used for ASNCs led to the formation of agglomerated spheres and irregular particles (Fig. 3A). Under the strongly acidic conditions, the interactions of protonated silicate species $\left(I^{+}\right)$, counterion $\left(\mathrm{X}^{-}\right)$, and SDA $\left(\mathrm{S}^{+}\right)$are perturbed by the protonated amines, leading to defects and the formation of an irregular particle morphology. This is in agreement with the previous observation that slight changes in the co-ion and counter-ion composition of the reaction mixture can have a significant effect on the morphology of the ASNCs. ${ }^{23}$

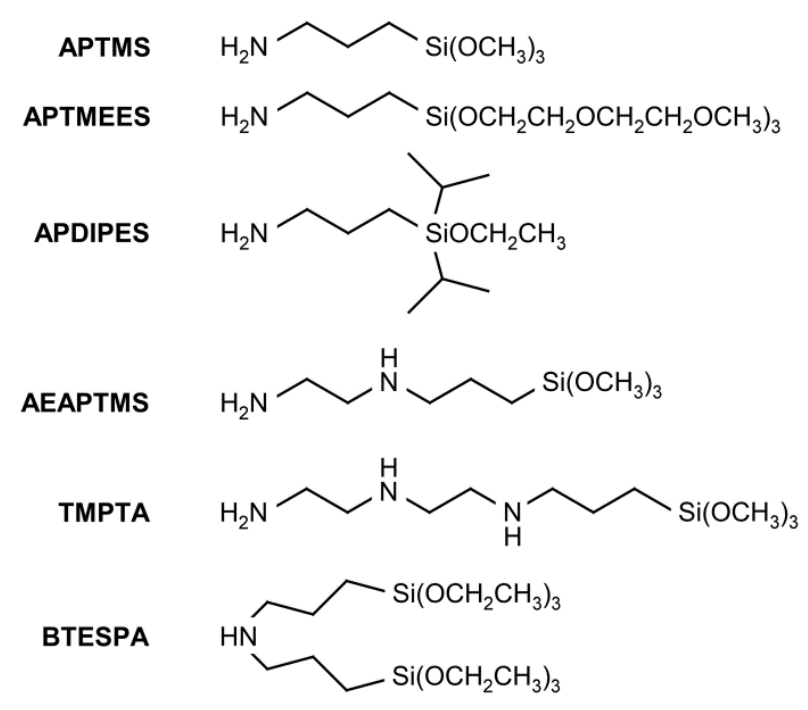

Fig. 2. Structures and abbreviations of amino-functional alkoxysilanes ( $\mathrm{NH}$-silanes) used in this work.

The addition of further TEOS after $3 \mathrm{~h}$ of reaction time produced a small amount of short hexagonal prismatic particles in addition to the ASNCs obtained in a conventional synthesis (Fig. $3 \mathrm{~B}$ and $3 \mathrm{C}$ ), 
indicating that free SDA in the reaction mixture is available for establishing $\mathrm{S}^{+} \mathrm{X}^{-} \mathrm{I}^{+}$interactions. This observation led us to the concept of post-condensation, i.e., adding an alkoxysilane to the reaction mixture after formation of the ASNCs.
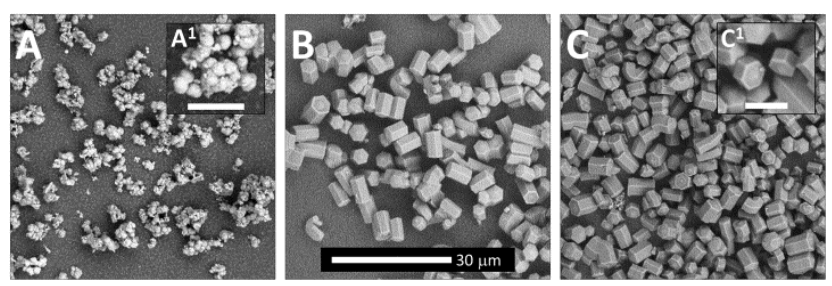

Fig. 3. SEM images of particles obtained by a co-condensation approach (APTMS/TEOS $=0.1$ ) under the conditions of the ASNCs synthesis (A). For comparison, pristine ASNCs obtained by a conventional synthesis (without the addition of APTMS) are shown (B). Post-condensation of TEOS leads to the formation of some small ASNCs, but otherwise has no significant effect on the particle morphology (C). The scale bars in $A^{1}$ and $C^{1}$ are $5 \mu \mathrm{m}$.

The addition of $\mathrm{NH}$-silanes after $3 \mathrm{~h}$ of reaction time did not affect the morphology of the ASNCs when the $\mathrm{NH}$-silane/TEOS ratio was kept below 0.3 (Fig. 4). The variation of the aspect ratio of aminofunctionalized ASNCs prepared with different $\mathrm{NH}$-silanes was in this case within the standard deviation range. At a NH-silane/TEOS ratio above 0.3 , an increase of the length of the particles was observed, accompanied by the appearance of particles with irregular morphology.

The post-condensation approach ensures a regular hexagonal prismatic morphology. The following processes might occur upon post-condensation: (i) Condensation of the NH-silane with the existing ASNCs particles, (ii) condensation of $\mathrm{NH}$-silanes (auto-condensation) or (iii) condensation of NH-silanes and free TEOS (co-condensation). The latter two reactions would lead to the formation of small irregular 
particles that might subsequently condense with the ASNCs. As we have not observed the formation of small by-product particles even at $\mathrm{NH}$-silane/TEOS ratios above 0.3 , the first process seems to be predominant.
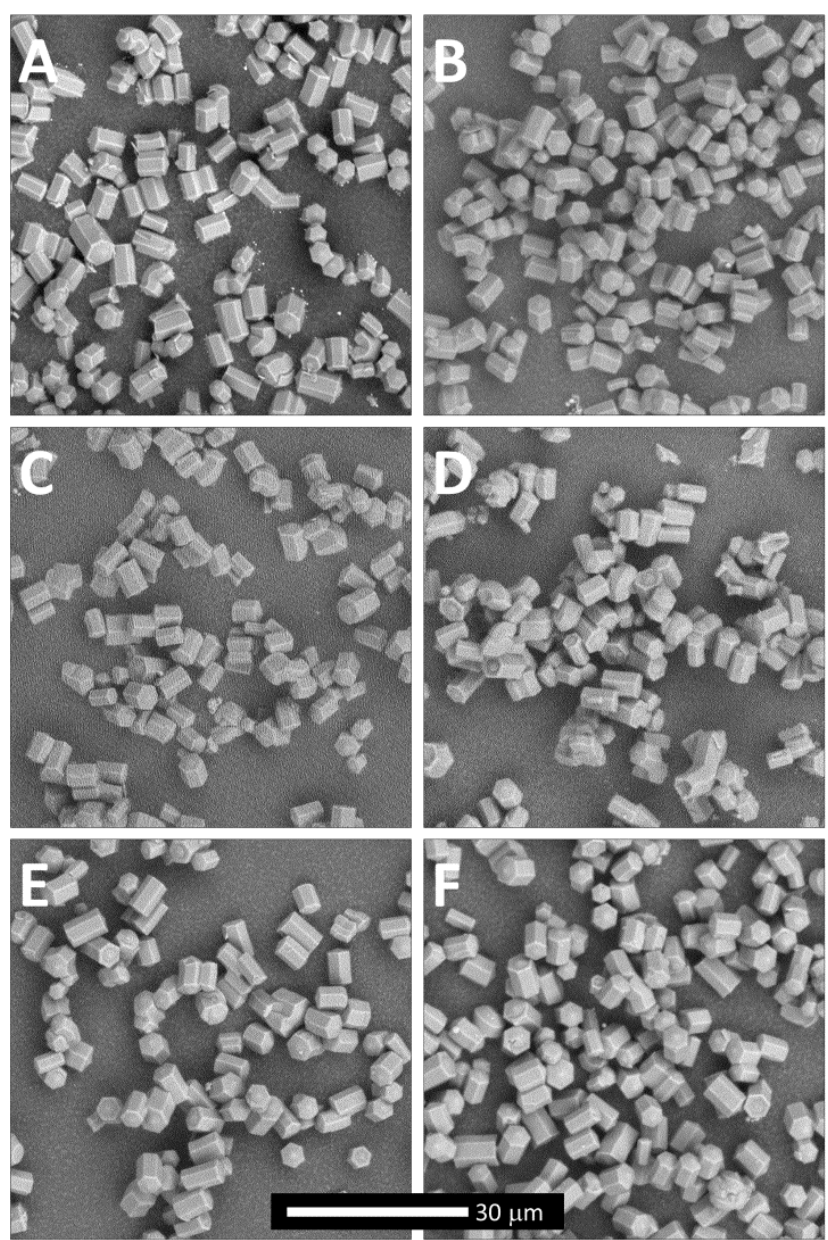

Fig. 4. SEM images of ASNCs synthesized by post-condensation with different NH-silanes (NHsilane/TEOS = 0.1): APTMS (A), AEAPTMS (B), TMPTA (C), APDIPES (D), BTESPA (E), APTMEES (F).

In highly acidic solutions, it has been observed that liquid-crystal embryos with hexagonal cross-sections develop into mesoporous silica fibers, leading to the conclusion that end-on growth plays a key role in 
the formation of the mesoporous silica framework under these conditions. ${ }^{37}$ Recent studies on the factors influencing the aspect ratio of ASNCs point to the importance of end-on growth in the formation of ASNCs. ${ }^{23}$ In the post-condensation of $\mathrm{NH}$-silanes, we can therefore expect that end-on growth directs the NH-silanes towards the hexagonal base surfaces of the ASNCs.

Pore structure. In order to preserve the amino groups in the ASNCs, the SDA needs to be removed under mild conditions. For MCM-41 type materials, efficient removal of alkyltrimethylammonium SDAs has been achieved by extraction with an ethanolic solution of ammonium nitrate. ${ }^{38}$ Experiments with pristine ASNCs revealed that applying this established procedure for the extraction of alkyltrimethylammonium SDAs led to a collapse of the ASNCs. As a consequence, a type I nitrogen adsorption isotherm and broad pore size distribution was obtained (Fig. 5, sample 3). To increase the stability of the silica framework prior to extraction, as-synthesized samples were treated at $150{ }^{\circ} \mathrm{C}$ for $10 \mathrm{~h}$. This thermal pretreatment yielded a material featuring the expected reversible type $\mathrm{IVb}$ isotherm ${ }^{39}$ and a narrow pore size distribution centered at $3.9 \mathrm{~nm}$ (Fig. 5, sample 1). The need for a thermal treatment before extraction is a consequence of the low temperature synthesis of ASNCs, which produces a silica framework with a low degree of cross-linking. A significant decrease of the pore size is therefore observed upon calcination (Fig. 5, sample 2).

Nitrogen sorption isotherms and pore size distributions of samples synthesized by post-condensation with $\mathrm{NH}$-silanes are shown in Fig. 6. The structural properties are summarized in Table 1. Comparison with the pristine extracted sample reveals that the $\mathrm{NH}$-silane post-condensation does not cause a significant change in the porosity. All samples feature a reversible type IVb isotherm. The similarity of the pristine and the amino-functionalized samples in terms of porosity is a consequence of postcondensation, i.e., formation of the ordered mesoporous structure prior to the addition of the NH-silane. 

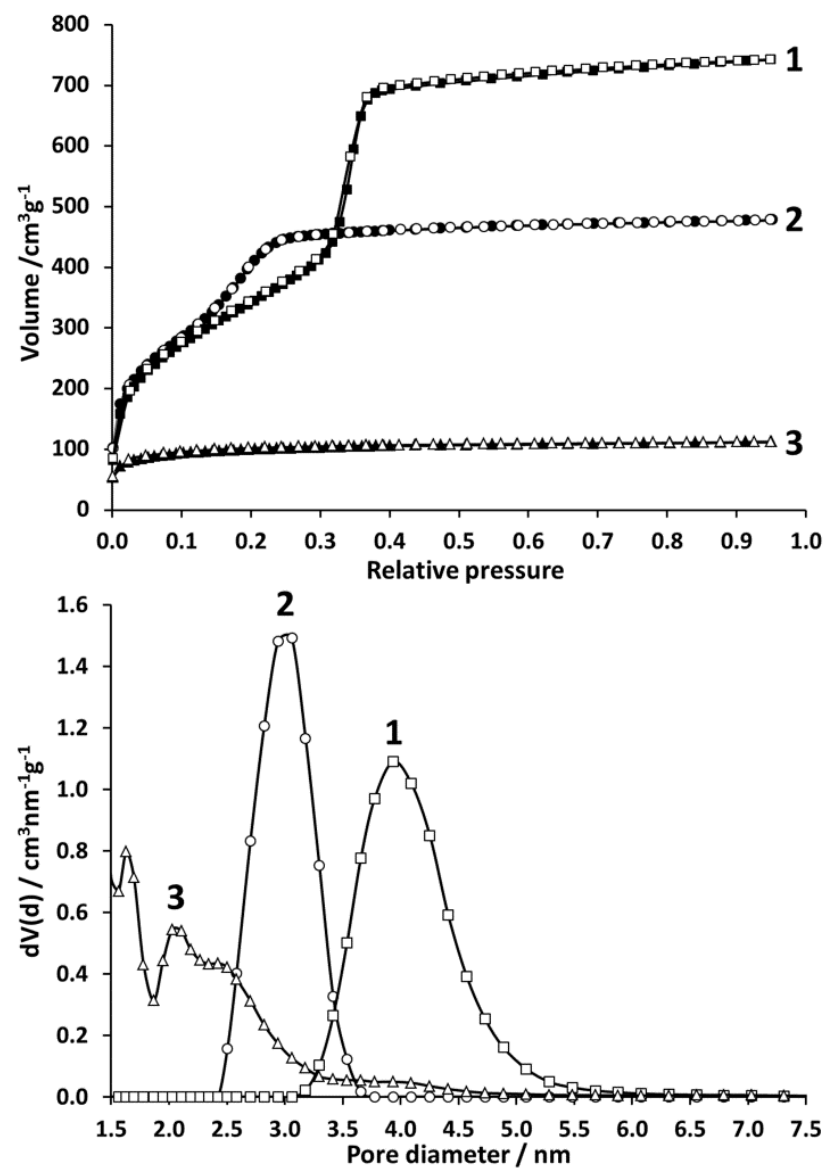

Fig. 5. Top: Nitrogen adsorption (filled symbols) and desorption (open symbols) isotherms of ASNCs after extraction with thermal pretreatment (1), calcination (2), and extraction without thermal pretreatment (3). Bottom: Respective pore size distributions. 
Table 1. Structural data of pristine and amino-functionalized ASNCs

\begin{tabular}{lcccc}
\hline NH-silane & $V_{\text {tot }} / \mathrm{cm}^{3} \mathrm{~g}^{-1}$ & $d_{\mathrm{DFT}} / \mathrm{nm}$ & $S_{\mathrm{BET}} / \mathrm{m}^{2} \mathrm{~g}^{-1}$ & $a_{0} / \mathrm{nm}^{\mathrm{c}}$ \\
\hline Pristine $(\mathrm{c})^{\mathrm{b}}$ & 0.7 & 3.1 & 1406 & $3.72(4.71)$ \\
Pristine & 1.1 & 3.9 & 1325 & $4.57(4.71)$ \\
APTMS & 0.9 & 3.9 & 1087 & $4.45(4.71)$ \\
APTMEES & 1.2 & 4.1 & 1162 & $4.54(4.70)$ \\
APDIPES & 1.2 & 4.1 & 1123 & $4.51(4.71)$ \\
AEAPTMS & 1.2 & 3.9 & 1421 & $4.48(4.70)$ \\
TMPTA & 1.1 & 3.9 & 1349 & $4.48(4.68)$ \\
BTESPA & 1.1 & 3.9 & 1057 & $4.54(4.67)$ \\
\hline
\end{tabular}

${ }^{a} \mathrm{NH}$-silane/TEOS $=0.1 .{ }^{\mathrm{b}}$ Calcined. ${ }^{\mathrm{c}}$ Values in parentheses are the repeat distances of the as-synthesized samples.
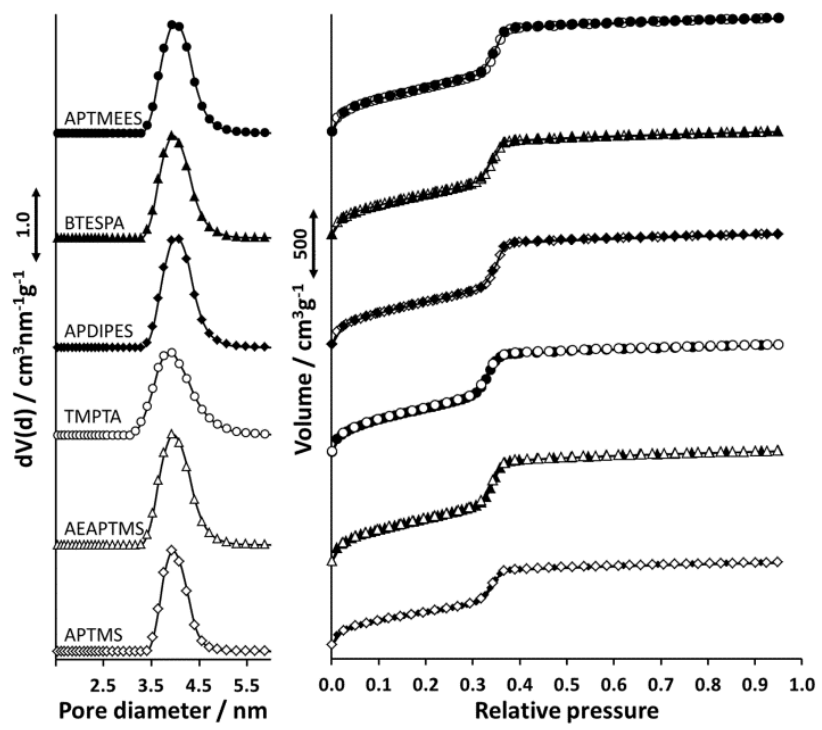

Fig. 6. Left: Pore size distributions of amino-functionalized ASNCs prepared by post-condensation and subsequent extraction of the SDA. The abbreviations indicate the respective NH-silane used in the postcondensation $(\mathrm{NH}$-silane/TEOS $=0.1)$. Right: Corresponding nitrogen sorption isotherms. 
XRD patterns of the pristine ASNCs are shown in Fig. 7. Representative patterns of the aminofunctionalized samples are summarized in Fig. 8. All samples feature the typical (100), (110), and (200) reflexes of the 2D hexagonal space group $\mathrm{p} 6 \mathrm{~mm}$. In agreement with the reduced pore diameter, calcination leads to a decreased repeat distance $a_{0}$, indicating a contraction of the silica framework due to further condensation.

Thermal treatment of the pristine as-synthesized sample before extraction causes framework stabilization by condensation, which manifests itself in a slightly decreased repeat distance compared to the respective as-synthesized sample. This effect is also observed when comparing the as-synthesized and extracted amino-functionalized samples (Table 1).

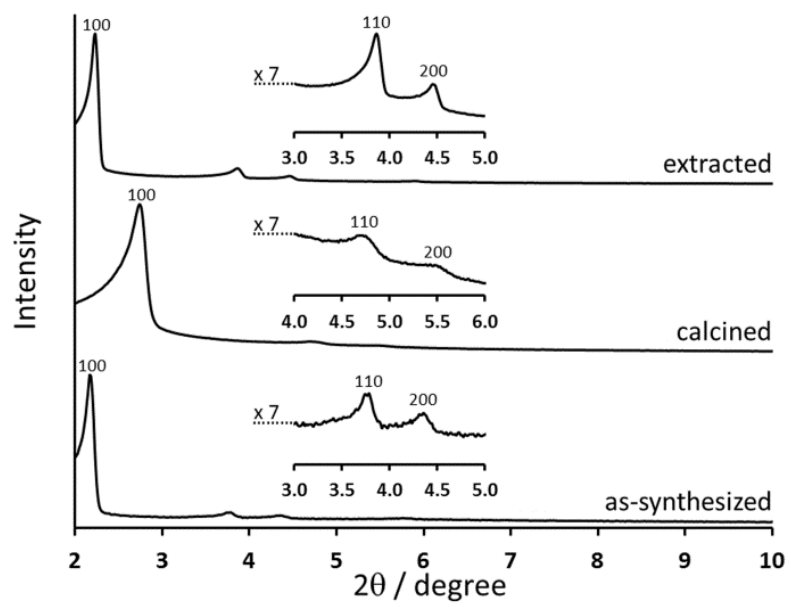

Fig. 7. XRD patterns of pristine ASNCs. The extracted sample was pretreated at $150^{\circ} \mathrm{C}$ before extraction. 


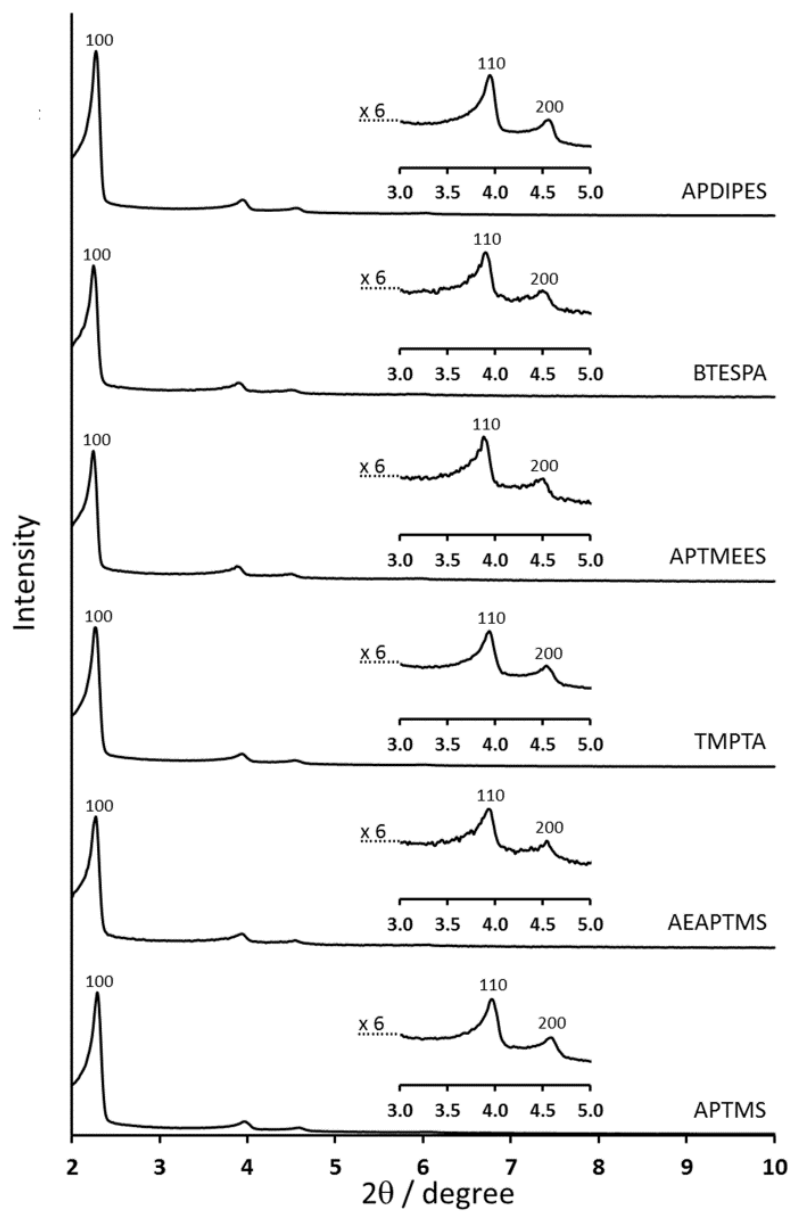

Fig. 8. XRD patterns of amino-functionalized ASNCs prepared by post-condensation and subsequent extraction of the SDA. The abbreviations indicate the respective $\mathrm{NH}$-silane used in the post-condensation (NH-silane/TEOS $=0.1$.

Distribution of amino groups. The spatial distribution of the amino groups was determined by CLSM after FITC coupling (Fig. 9). FITC coupling was conducted before (as-synthesized samples) or after (extracted samples) removal of the SDA. Comparison of the as-synthesized and extracted samples shows that the presence of the SDA does not affect the distribution of the fluorescein moieties after FITC coupling. However, the CLSM images of the extracted samples appeared brighter. This is most likely due 
to a better FITC coupling yield in the case of the extracted samples. No fluorescence was observed when applying the FITC coupling procedure to pristine ASNCs.

It has been shown that steric hindrance plays an important role in the postsynthetic functionalization of ASNCs with NH-silanes. ${ }^{10}$ Post-condensation is fundamentally different from postsynthetic functionalization. Introduction of the $\mathrm{NH}$-silane into the low $\mathrm{pH}$ reaction mixture leads to a fast hydrolysis of the alkoxysilane and protonation of the amino groups. We can therefore expect that in post-condensation, the size of the alkoxy groups does not influence the final distribution of the amino groups on the ASNCs and that the protonated NH-silanes can exchange the SDA in the ASNCs. This is best seen in the comparison of APTMS and APTMEES, which both led to a relatively uniform distribution of FITC-labeled amino groups despite differently sized alkoxy moieties (Fig. 9). In the case of APDIPES, a favorable interaction of the isopropyl moieties with the hydrophobic tail of the SDA might further support a uniform distribution. These results are in contrast to postsynthetic functionalization, where APTMEES can be used to generate a selective functionalization of the external surface. ${ }^{10}$

Unlike classical co-condensation, post-condensation can lead to non-uniform distributions of functional groups. In the case of AEAPTMS, TMPTA, and BTESPA, fluorescence is mainly observed at the base surfaces of the ASNCs, indicating a significant degree of channel entrance functionalization (Fig. 9). The accumulation of FITC-labeled amino groups at the channel entrances is particularly evident for samples synthesized with AEAPTMS and TMPTA. The comparatively large size of these molecules in combination with end-on growth ${ }^{37,40}$ is most likely responsible for the channel entrance accumulation. 


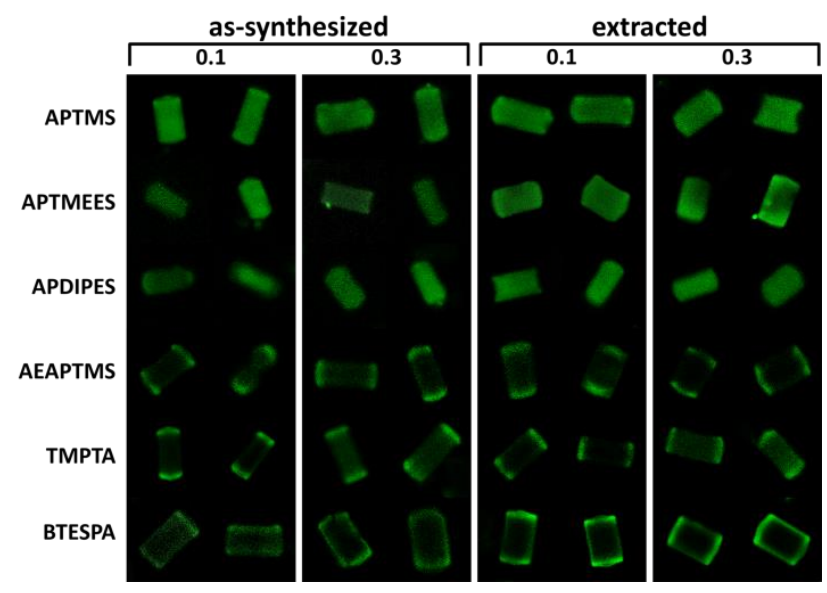

Fig. 9. CLSM images of FITC-labeled amino-functionalized ASNCs prepared by post-condensation. The columns contain images for as-synthesized and extracted samples produced with NH-silane/TEOS ratios of 0.1 and 0.3 . The respective $\mathrm{NH}$-silane is indicated on the left. Optical slices in the center of the particles were selected (excitation at $488 \mathrm{~nm}$ ).

Accessibility. The selective functionalization of the channel entrances is of interest for establishing concepts of controlled release, e.g. via the grafting of molecular gates. ${ }^{41}$ Methods for the selective functionalization of channel entrances are only useful if the channels and thus the pore surface remain accessible. To test the accessibility of channel entrance functionalized samples, we have conducted FITC coupling, followed by postsynthetic functionalization with APTMS and labeling with Texas Red (TR). The corresponding CLSM images indicate that the TR labels have entered the nanochannels, despite the presence of the fluorescein labels at the entrances (Fig. 10).

An interesting effect was observed in the FITC/TR-labeled samples. For samples prepared with AEAPTMS, the TR labels are distributed rather uniformly throughout the material. This is best seen in the images B of Fig. 10, which were acquired by excitation at $543.5 \mathrm{~nm}$, where the absorption of the fluorescein labels is negligible, and observation of the TR fluorescence. However, excitation at $488 \mathrm{~nm}$ and observation of 
the TR fluorescence reveals an increased intensity at the channel entrances (images C, Fig. 10). As the absorption of TR is negligible at $488 \mathrm{~nm}$, the increased TR fluorescence is most likely caused by energy transfer from the fluorescein labels located at the channel entrances. A similar effect was observed for the samples prepared with TMPTA. When inspecting several of the FITC/TR-labeled particles, we noticed instances of non-uniform TR distributions, as shown in the lower left panel of Fig. 10. Selective observation of the TR labels indicates an accumulation of TR at the channel entrances, which could be the result of pores partially blocked by the large TMPTA-FITC conjugates.

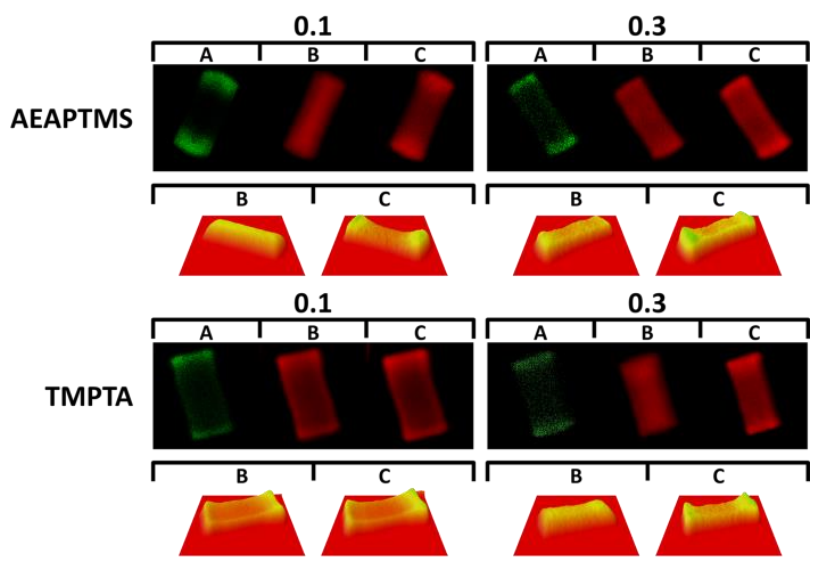

Fig. 10. CLSM images of FITC-labeled amino-functionalized ASNCs prepared by post-condensation (NHsilane/TEOS ratio 0.1 or 0.3 ) and subsequent postsynthetic modification with APTMS and TR-labeling. A) Excitation at $488 \mathrm{~nm}$ and observation of FITC fluorescence. B) Excitation at $543.5 \mathrm{~nm}$ and observation of TR fluorescence. C) Excitation at $488 \mathrm{~nm}$ and observation of TR fluorescence. The distribution of the fluorescence intensity in the images B and C is also given by the corresponding intensity profiles (red: low intensity, yellow: intermediate intensity, green: high intensity). Optical slices in the center of the particles were selected. 


\section{Conclusions}

The combination of the established mesoporous silica functionalization pathways, namely postsynthetic functionalization and co-condensation, eliminates some of the drawbacks of these two methods. Postsynthetic functionalization favours the most accessible sites on the external particle surface, whereas co-condensation is unable to selectively modify different surface regions.

The preparation of amino-functionalized ASNCs by post-condensation preserves the well-defined hexagonal prismatic particle morphology and narrow pore size distribution. The distribution of the amino groups in the ASNCs can be controlled by the type of amino-functional alkoxysilane. Molecules that are sterically hindered after hydrolysis in the low $\mathrm{pH}$ reaction mixture tend to accumulate at the channel entrances. Dual fluorescent labeling experiments and CLSM imaging revealed that the nanochannels of post-condensed ASNCs are accessible for further postsynthetic functionalization steps. This opens possibilities for the preparation of multifunctional mesoporous particles with defined functional group distributions. The selective functionalization of the channel entrances is expected to provide a starting point for introducing stimuli-responsive molecular gates ${ }^{41}$ or for establishing a vertical orientation of ASNCs on a flat surface as well as specific particle-particle interactions, similar to what has been achieved for microporous materials such as zeolite L. $^{42-44}$

\section{Acknowledgements}

Financial support by the Swiss National Science Foundation (project 200021 149715) is acknowledged. The authors thank Dr. Fabio Evangelisti, Michael Olah, and Prof. Dr. Greta Patzke for XRD measurements. 


\section{References}

1 L.-B. Sun, X.-Q. Liu and H.-C. Zhou, Chem. Soc. Rev., 2015, 44, 5092-5147.

2 S. Rostamnia and E. Doustkhah, RSC Adv., 2014, 4, 28238-28248.

3 X. Qian, K. Fuku, Y. Kuwahara, T. Kamegawa, K. Mori and H. Yamashita, ChemSusChem, 2014, 7, 1528-1536.

4 F. Sancenón, L. Pascual, M. Oroval, E. Aznar and R. Martinez-Máñez, ChemistryOpen, 2015, 4, 418-437.

5 S. Alberti, G. J. A. A. Soler-Illia and O. Azzaroni, Chem. Commun., 2015, 51, 6050-6075.

6 A. Walcarius and L. Mercier, J. Mater. Chem., 2010, 20, 4478-4511.

7 F. Hoffmann, M. Cornelius, J. Morell and M. Fröba, Angew. Chem. Int. Ed., 2006, 45, 3216-3251.

8 D. S. Shephard, W. Zhou, T. Maschmeyer, J. M. Matters, C. L. Roper, S. Parsons, B. F. G. Johnson and M. J. Duer, Angew. Chem. Int. Ed., 1998, 37, 2719-2723.

$9 \quad$ K. Cheng and C. C. Landry, J. Am. Chem. Soc., 2007, 129, 9674-9685.

10 N. Gartmann and D. Brühwiler, Angew. Chem. Int. Ed., 2009, 48, 6354-6356.

11 Y. Huang, S. Xu and V. S.-Y. Lin, Angew. Chem. Int. Ed., 2011, 50, 661-664.

12 J. D. Webb, T. Seki, J. F. Goldston, M. Pruski and C. M. Crudden, Microporous Mesoporous Mater., 2015, 203, 123131.

13 S. Huh, J. W. Wiench, J.-C. Yoo, M. Pruski and V. S.-Y. Lin, Chem. Mater., 2003, 15, 4247-4256.

14 S. Sadasivan, D. Khushalani and S. Mann, J. Mater. Chem., 2003, 13, 1023-1029.

15 J. L. Blin, C. Gérardin, L. Rodehüser, C. Selve and M. J. Stébé, Chem. Mater., 2004, 16, 5071-5080.

16 C. Yang, Y. Wang, B. Zibrowius and F. Schüth, Phys. Chem. Chem. Phys., 2004, 6, 2461-2467.

17 R. P. Hodgkins, A. E. Garcia-Bennett and P. A. Wright, Microporous Mesoporous Mater., 2005, 79, $241-252$.

18 K. Möller, J. Kobler and T. Bein, J. Mater. Chem., 2007, 17, 624-631.

19 F. O. M. Gaslain, C. Delacôte, A. Walcarius and B. Lebeau, J. Sol-Gel Sci. Technol., 2009, 49, 112-124.

20 Y. Kievsky and I. Sokolov, IEEE Trans. Nanotechnol., 2005, 4, 490-494.

21 D. O. Volkov, J. Benson, Y. Y. Kievsky and I. Sokolov, Phys. Chem. Chem. Phys., 2010, 12, 341-344.

22 N. Gartmann, C. Schütze, H. Ritter and D. Brühwiler, J. Phys. Chem. Lett., 2010, 1, 379-382.

23 N. Zucchetto and D. Brühwiler, RSC Adv., 2015, 5, 74638-74644.

24 A. Zabala Ruiz, D. Brühwiler, T. Ban and G. Calzaferri, Monatsh. Chem., 2005, 136, 77-89.

25 A. G. Gomez, G. de Silveira, H. Doan and C.-H. Cheng, Chem. Commun., 2011, 47, 5876-5878.

26 A. I. Lupulescu, M. Kumar and J. D. Rimer, J. Am. Chem. Soc., 2013, 135, 6608-6617. 
27 T. Kodaira, K. Miyazawa, T. Ikeda and Y. Kiyozumi, Microporous Mesoporous Mater., 1999, 29, 329-337.

28 F. Y. Jiang, Z. K. Tang, J. P. Zhai, J. T. Ye and J. R. Han, Microporous Mesoporous Mater., 2006, 92, 129-133.

29 Z. Liu, T. Wakihara, D. Nishioka, K. Oshima, T. Takewaki and T. Okubo, Chem. Mater., 2014, 26, $2327-2331$.

30 D. Brühwiler, G. Calzaferri, T. Torres, J. H. Ramm, N. Gartmann, L.-Q. Dieu, I. Lopez-Duarte and M. V. Martinez-Diaz, J. Mater. Chem., 2009, 19, 8040-8067.

31 P. Cao, O. Khorev, A. Devaux, L. Sägesser, A. Kunzmann, A. Ecker, R. Häner, D. Brühwiler, G. Calzaferri and P. Belser, Chem. Eur. J., 2016, 22, 4046-4060.

32 G. Ihlein, F. Schüth, O. Krauss, U. Vietze and F. Laeri, Adv. Mater., 1998, 10, 1117-1119.

33 F. Y. Jiang, W. X. Lu, J. P. Zhai, J. T. Ye, G. K. L. Wong, J. R. Han and Z. K. Tang, J. Phys. Chem. B, 2006, 110, 8924-8927.

34 N. Gartmann and D. Brühwiler, Materials, 2011, 4, 1096-1103.

35 J. Landers, G. Y. Gor and A. V. Neimark, Colloid. Surf. A: Physicochem. Eng. Aspects, 2013, 437, 3-32.

36 S. Brunauer, P. H. Emmett and E. Teller, J. Am. Chem. Soc., 1938, 60, 309-319.

37 H. Yang, N. Coombs and G. A. Ozin, Nature, 1997, 386, 692-695.

38 N. Lang and A. Tuel, Chem. Mater., 2004, 16, 1961-1966.

39 M. Thommes, K. Kaneko, A. V. Neimark, J. P. Olivier, F. Rodriguez-Reinoso, J. Rouquerol and K. S. W. Sing, Pure Appl. Chem., 2015, 87, 1051-1069.

40 G. A. Ozin, C. T. Kresge and H. Yang, Stud. Surf. Sci. Catal., 1998, 117, 119-127.

41 E. Aznar, M. Oroval, L. Pascual, J. R. Murguia, R. Martinez-Máñez and F. Sancenón, Chem. Rev., 2016, 116, 561-718.

42 A. Zabala Ruiz, H. Li and G. Calzaferri, Angew. Chem. Int. Ed. 2006, 45, 5282-5287.

43 J. S. Lee, H. Lim, K. Ha, H. Cheong and K. B. Yoon, Angew. Chem. Int. Ed. 2006, 45, 5288-5292.

44 Z. Popović, M. Busby, S. Huber, G. Calzaferri and L. De Cola, Angew. Chem. Int. Ed. 2007, 46, 8898-8902. 